\title{
Shape Control of Electronic Devices by Soldering
}

\author{
Hiroshi Kikuchi*, Norio Nakazato**, Naotaka Tanaka** and Toshihiko Sato*** \\ *Micro Device Division, Hitachi, Ltd., 6-16-3, Shinmachi Ome-shi, Tokyo, 198-8512 Japan \\ **Mechanical Engineering Research Laboratory, Hitachi, Ltd., 832-2 Horiguchi, Hitachinaka-shi, 312-0034 Japan \\ ***Packaging \& Test Technology Div., LSI Manufacturing Technology Unit, Renesas Technology Corp., 5-20-1 Jousuihon-cho, Kodaira-shi, Tokyo, 187- \\ 8588 Japan
}

(Received July 5, 2009; accepted October 20, 2009)

\begin{abstract}
The authors investigated shape-control technology for electronic devices using the material characteristics of solder. Specifically, the authors developed and put into practical use the following processes and structures: (1) A "Solder bump transfer process" for reducing variation in the solder bump heights. (2) A "Support ball structure”, by which high-meltingpoint solder spacers are set on the four corners of a BGA to control the heights of solder balls. (3) A "Solder pouring process", which allows voidless soldering with molten solder poured into metallized parts on electronic devices. We found that the support ball method is effective for heavy BGA structure packages used in high-speed communication. A highquality hermetic sealing package was realized by application of the solder pouring method.
\end{abstract}

Keywords: Solder Bump, Transfer Process, Parallel Plates, Support Ball, Pouring Process

\section{Introduction}

In semiconductor implementations, solder is used for electric contact points, heat dissipation, sealing, etc. In common soldering techniques, solid solder is set on the metal to be soldered. Then the solder is reflowed during the oxide film removal of solder with flux. The key in soldering is solder's wettability for metallization. With insufficient wettability, the junction area or bonding strength might be reduced, thereby causing soldering defects. Therefore, in soldering, it is necessary to secure the quality of the soldering materials themselves as well as that of the metallization.

In the soldering process, it is possible to use the characteristics of molten solder along with the characteristics of solder as a solid, and to control, with high precision, the height of a soldering electrode and shapes of electronic devices, using differences in the solder wettability of the metal and other parts. Here, the authors report on (1) a technique to planarize plural solder contact points (Section 2) and (2) a technique to solder in a specified dimension (Section 3). Furthermore, the authors describe the applied technology using basic techniques.

\section{Technique to Planarize Plural Solder Contact Points}

\subsection{Study purpose}

As the integration and speed of LSI progress, it is necessary to increase the number of pins for packaging technology.[1,2] When solder bumps are formed on a semiconductor chip, processes such as printing in the form of a wafer, plating, ball supply, and vacuum evaporation are usually performed. Therefore, the processing time of the product wafer that forms a device increases for each additional solder bump formation process. Furthermore, solder bumps are formed on the entire wafer surface. Bumps are formed on all chips, irrespective of whether the wafer is electrically non-defective or defective.

Several hundred to several thousand solder bumps are formed on an IC chip. However, if the solder bump height varies, soldering defects might occur in reflow. As a countermeasure, all bumps are examined after bump formation. In some cases, however, this increases the labor necessary for production. Therefore, in this study, the authors equalized the solder bump height and examined a process by which solder bumps are formed only on non-defective chips.

\subsection{Solder bump transfer process}

Figure 1 presents an illustration of the solder-bump 


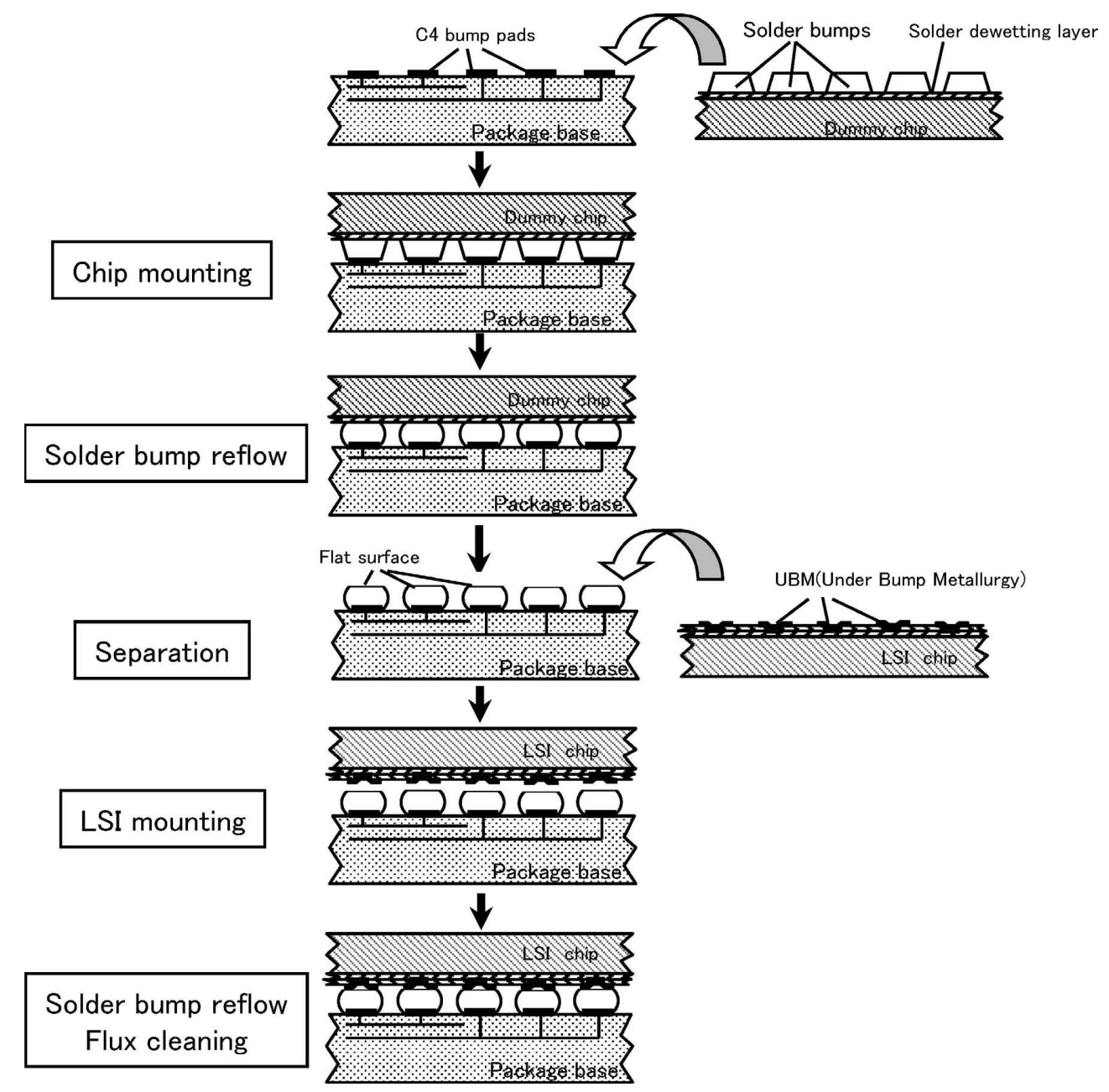

Fig. 1 Solder bump transfer process.

transfer process developed in this study.[3] Solder bumps are formed on a dummy chip with material that is difficult to wet with solder on its surface. After applying flux on the solder bumps, they were set opposite to the metallized base. Furthermore, the solder was reflowed. After reflowing, the dummy chip and base were separated and the flux was removed. At this stage, the solder bumps were transferred onto the metallized base.

Next, a non-defective LSI chip was set on the base with solder bumps and was ready after being reflowed and cleaned. As for variation of bump heights when the solder bump-transfer process is used, high precision can be realized because, in principle, it is nearly equivalent to the planarization grade of the dummy chip (less than several micrometers). In addition, solder bumps are formed only on non-defective chips. For that reason, reduced solder material loss is a characteristic of this process.

\subsection{Technical problem}

The solder-bump transfer process transfers solder bumps from a dummy chip with a surface that is difficult to wet with solder to the metallized base, which is easy to wet with solder. When reflowing solder, the solder is dewetted on a face that is hard to wet with solder; it instead wets on the metallizing base. Solder moves by this interaction.

An important problem occurs when proper self-alignment is obtained between the upper and lower sides of the base: the solder wets on one side and is de-wetted on the other side simultaneously. This description applies to the situation shown in Figure 2. The positions of the LSI chip and package base were determined using a chip mounter and set. Positioning errors of around several dozen micrometers might occur because of variations in the chip mounter and metallization patterns. In the reflow process, molten solder wets both the LSI chip and the package base. However, the chip position is corrected with the surface tension of solder bumps. To a certain degree, even if there are displacement errors, through this self-alignment it is possible to set the upper and lower parts in the correct positions without causing a short circuit between bumps.

For the solder-bump transfer process introduced in this study, a dummy chip with a face that is difficult to wet with 

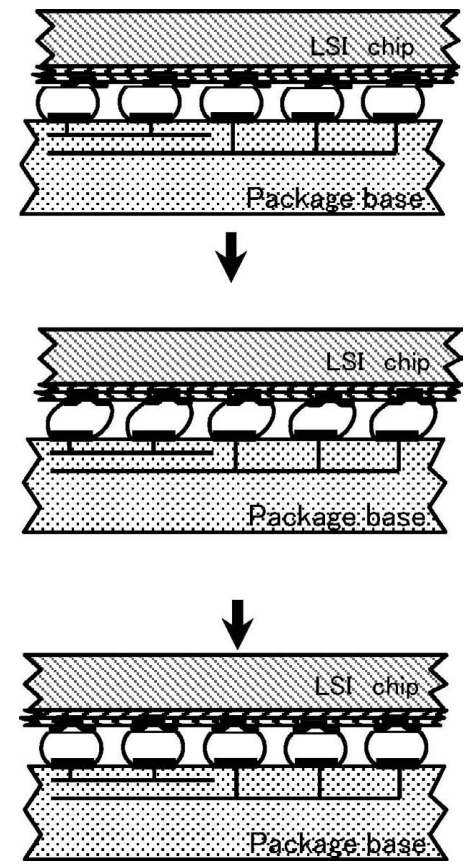

Fig. 2 Schematic of the self-alignment process.

solder is used. The point of the experiment is to see if the dummy chip moves significantly in reflow and causes a bump short.

\subsection{Result of experimental manufacture}

Figure 3 depicts the observed results of solder bumps on the dummy chip. The dummy chip was created by dicing silicon wafers formed with silicon oxides and solder bumps on them. The solder bumps were Pb-Sn Alloy. After applying flux on the dummy chip with solder bumps, the dummy chip was placed opposite the base with metallic pad faces. They were separated after reflow. Figure 4 shows the base and the dummy chip after separation. All the solder bumps on the dummy chip are transferred onto the base. For this experimental manufacture, in the reflow, the dummy chip did not move greatly and the solder bumps did not short out as had initially been foreseen.

\subsection{Analysis of experimental manufacture}

Figure 5 shows solder bumps formed on the dummy chip. Figure 6 shows SEM observation examples after the transfer.

Multiple small solder grains remain in a ring on the dummy chip after the transfer of solder bumps. These parts, which have solder grains, show the spots where the solder bumps had been before the transfer. In addition, traces of round solder bumps are apparent in the central region of the solder grains. This is the spot where the solder bump tips contact the dummy chip surface, even after the reflow.

Figure 7 shows SEM observation images after comple-

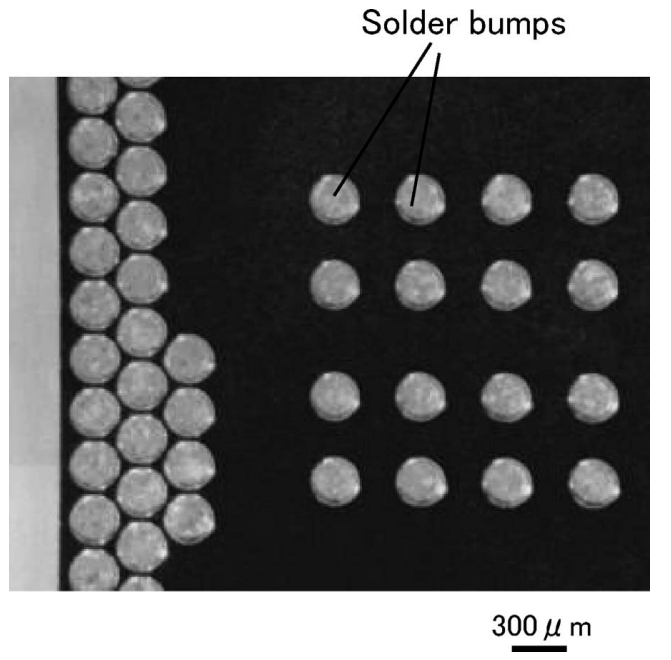

Fig. 3 Solder bumps on the dummy chip.

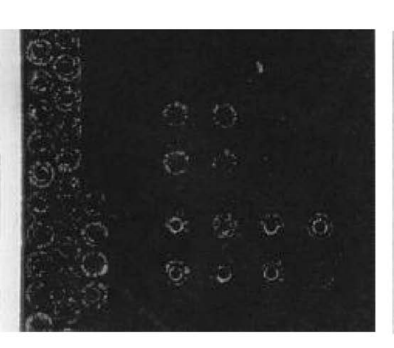

Surface image of the dummy chip

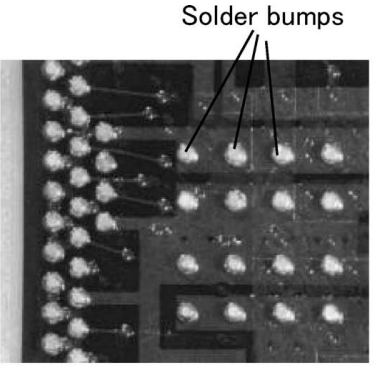

Surface image of the package base $300 \mu \mathrm{m}$
Fig. 4 After bump transfer process.

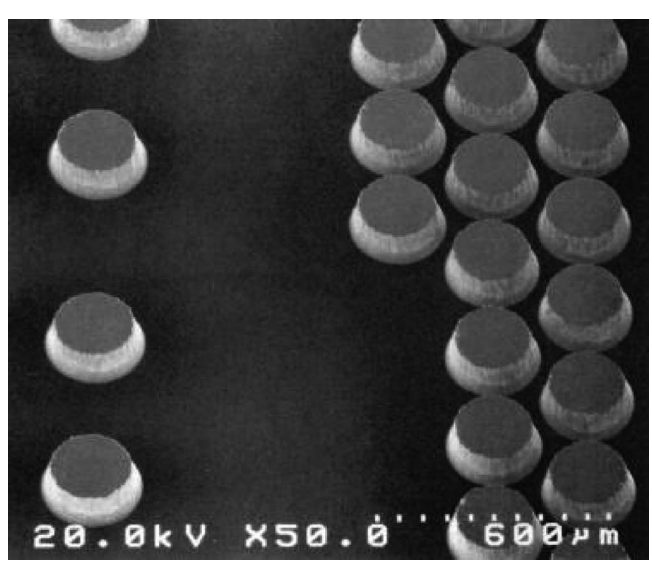

Fig. 5 Solder bumps on the dummy chip.

tion of the transfer. The bumps have flat parts of about $\varnothing 80 \mu \mathrm{m}$. These parts correspond to the trace of contact with solder bumps presented in Figure 6.

From the analysis result described above, the solder bumps on the dummy chip are de-wetted by the reflow and become spheres. However, the bump tips were planarized with the weight of the dummy chip. This phenomenon is useful for implementation of an LSI chip having solder bumps. 


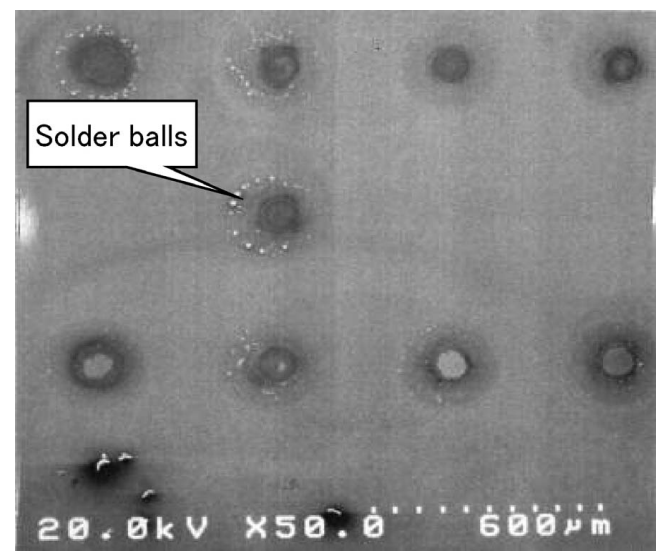

Fig. 6 Surface image of the dummy chip (After bump transfer process).

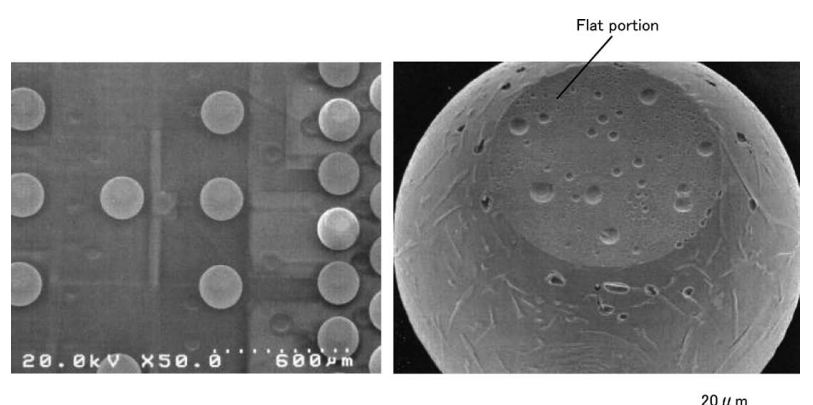

Fig. 7 SEM image of solder bumps (After bump transfer process).

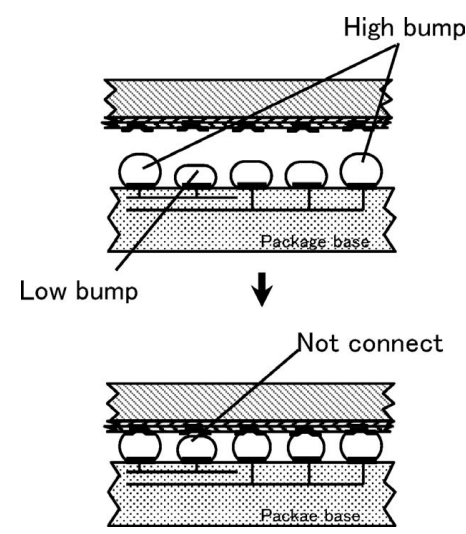

Fig. 8 Schematic diagram of bump connection poor.

Practically speaking, the solder bump heights must be uniform for the connection of solder bumps. For instance, as presented in Figure 8, for the case in which the heights of solder bumps vary, non-connections occur on low solder bumps. In the solder-bump transfer process used this study, the solder bump tips are planarized using the dummy chip. Using this principle, the solder bump height variation was equal to the planarization level of the dummy chip. The dummy chip was manufactured from a silicon wafer for semiconductors. For that reason, the planarization level was extremely low. Furthermore, even when
Table 1 Success rate of bump transfer.

\begin{tabular}{ll}
\hline \multicolumn{1}{c}{ Type } & \multicolumn{1}{c}{ Sucsess rate } \\
\hline Silicon wafer & Less than $5 \%$ \\
Heat oxidized film on Si chip & About $20 \%$ \\
Cr film on Si chip & More than $99 \%$ \\
\hline
\end{tabular}

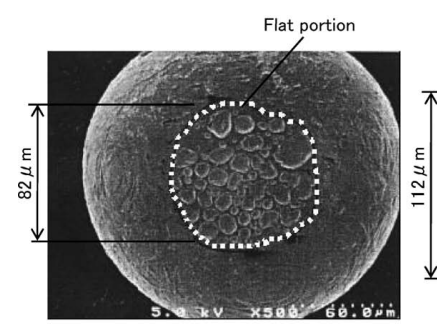

Using $\mathrm{Cr}$ film on Si chip

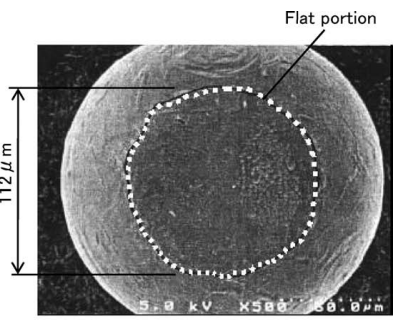

Using heat oxidized film on Si chip
Fig. 9 SEM image of solder bumps (After bump transfer process).

irregularities on the base surface occur with the transferred solder bumps, the solder bumps are planarized by the dummy chip. Therefore, it is possible to reduce the non-connection potential attributable to solder bump height variation when a LSI chip is set on a base package. In addition, as shown by the bump marks portrayed in Figure 6 , the reflow of the solder bumps was completed with the connection force for the dummy chip.

Table 1 presents an evaluation of the success rates of solder bump transfers for various dummy chip specifications. Silicon chips with oxide films and chips with chromium films were used. When a transfer succeeded, all bumps were transferred onto the base. When a transfer failed, solder bumps that were not transferred remained on the dummy chip.

The results of the experiment show that it is difficult to separate the components after transfer with a silicon chip. However, an excellent result was obtained for chips with chromium films.

Figure 9 presents bump observation examples for the cases in which chips with chromium films and chips with silicon oxides were used as dummy chips. The collapsed areas on the bump tips are smaller in the chips with chromium films than in the chips with silicon oxides. The authors presume that the wettability of chromium films is less than those of other materials, and for that reason, the connection area is small. Thus, chromium films were separated easily from the solder.

As solder bumps are miniaturized further in the future, it will be increasingly necessary to reduce the variability of solder bump heights to achieve better connections. In 
such a case, we cannot ignore the influences of irregularities and warps of the base surface. The authors infer that it is possible to keep the variation of solder bump heights low through application of the solder-bump transfer process used for this study method. In doing so, connection yields can be improved.

\section{Technique to Solder in Desired Dimension}

\subsection{Height control of BGA solder ball}

\subsubsection{Purpose}

In recent years, semiconductor chips have become larger as high integration and multifunctionalization of semiconductor progresses. Moreover, the planar sizes of the package bases on which semiconductor chips are installed are becoming larger. Furthermore, calorific values have increased with the increasing speed of loop systems equipped on semiconductor chips. For that reason, large heat sinks are typically installed to cool semiconductor chips.

When a Ball Grid Array (BGA) structure is adopted with such large and heavy packages, connection heights become low when the loads added to the solder ball increase. Furthermore, when the loads increase beyond a certain point, the solder balls short-circuit.

From another viewpoint, in packages of BGA structures, it is necessary to ensure a certain height of the solder balls to reduce thermal strains occurring between the package and the motherboard on which the package is mounted. The connection life of the polar zones shortens if the package loads increase and the solder ball height becomes lower.

For these reasons, solder balls of BGA structures are best when fixed, irrespective of the semiconductor package's weight. The authors studied a method for controlling the heights of solder balls with BGA structures.

\subsubsection{Support ball structure}

As described above, in a BGA structure package, the connection heights are reduced when the package loads increase. Furthermore, when the loads increase beyond a certain limit, the solder balls short-circuit.

As shown in Figure 10, for a BGA having 359 solder balls mounted on a motherboard, when the load increased, a short circuit occurred among solder balls with approximately $20 \mathrm{~g}$ load, as shown in Figure 11. Figure 12 presents transmission X-ray observation images of shortcircuited solder balls.

As a method to control the solder ball height, core spheres are sometimes inserted. For example, copper or

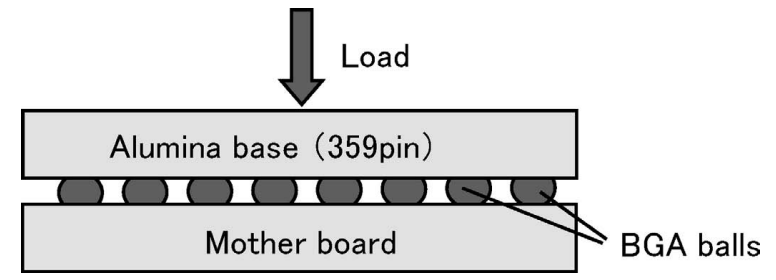

Fig. 10 Illustration of the BGA sample.

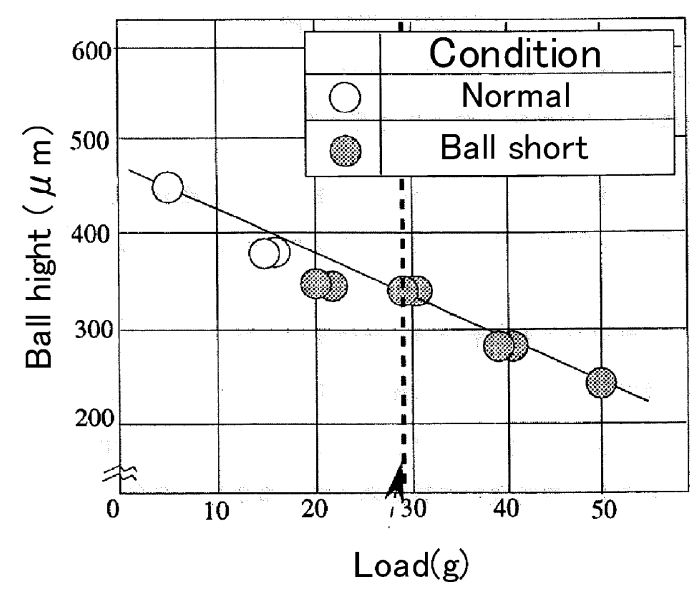

Fig. 11 Relationship between load and ball height.

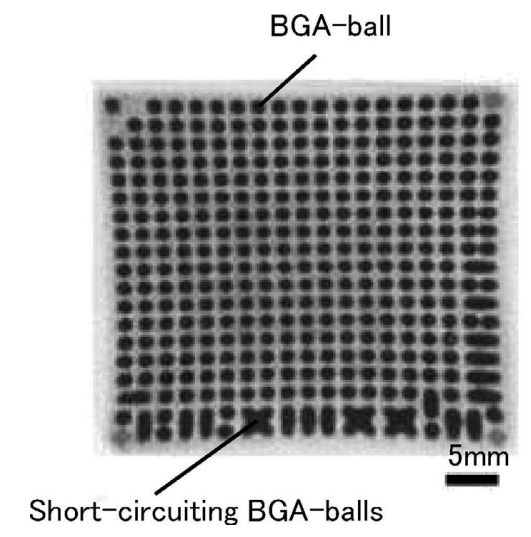

Fig. 12 Transmission X-ray image of BGA-balls.

plastic balls are used as cores and are wrapped with solder material.

Using this method, when it becomes necessary to change a BGA structure package after mounting it on a motherboard, the solder balls remain on electrodes of the base or motherboard after removing the package. Therefore, it is necessary to remove the remaining solder balls before remounting the package. To avoid this problem, in this study, the authors examined a solder-ball height control method without cores for solder balls.

Figure 13 portrays illustrations of the support ball method used in this study. High-melting-point solder balls are formed at the corners of a BGA base. The heights of the high-melting-point solder balls are determined by the electrode areas and the volume of solder balls to be 


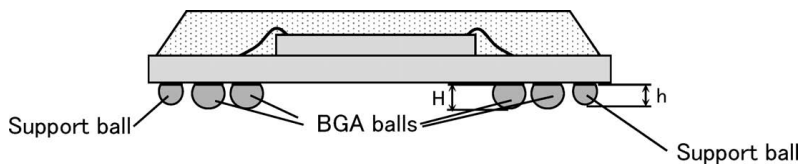

Fig. 13 Schematic diagram of the semiconductor-package with support balls.

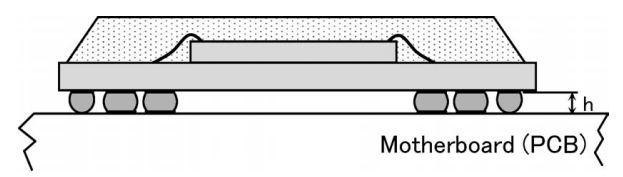

Fig. 14 Schematic diagram of the semiconductor-package on motherboard.

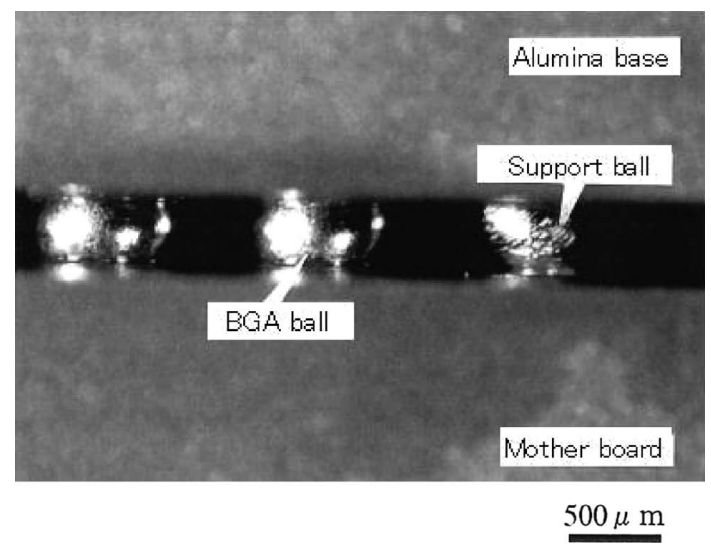

Fig. 15 Side view of the package.

poured. The heights of the high-melting-point solder balls are designed to be those of connections obtained when a package is mounted on the motherboard. In addition, lowmelting-point solder balls are formed on the BGA polar zone except at the corners.

Figure 14 shows illustrations of this package mounted on the motherboard. During the reflow process, in which the package is mounted on the motherboard, the low-melting-point solder balls melt first. The connection heights decrease with the package weight. However, the highmelting-point support balls can act as a support and prevent the collapse of the low-melting-point solder balls.

Figure 15 shows side observation images in which support balls are set on the corners of the sample with 359 pins, as shown in Figure 10; then reflow is performed. The figure shows that the high-melting-point support balls support low-melting-point balls.

Figure 16 shows the connection heights obtained through variation of the loads. The connection heights of the BGA are constant with support balls, even when the loads increase.

Figure 17 contains transmission X-ray observation images for a $50 \mathrm{~g}$ load. There is no solder ball collapse.

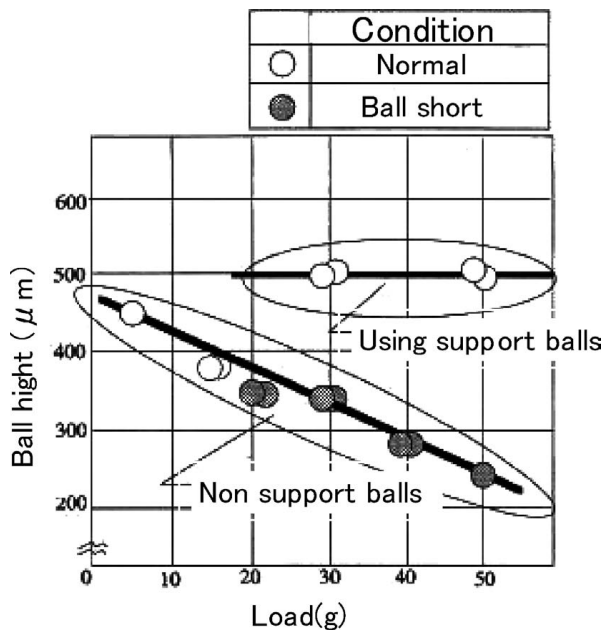

Fig. 16 Relationship between load and ball height.

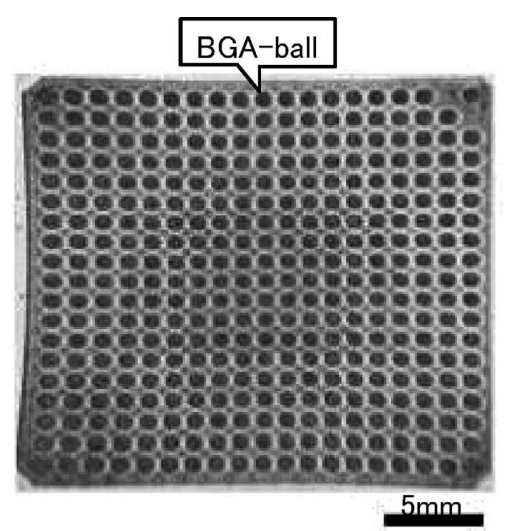

Fig. 17 Transmission X-ray image of BGA-balls.

One characteristics of this support ball method is that the support ball heights are less than those of the low-meltingpoint solder balls. When the low-melting-point solder balls melted, self-alignment of the upper and lower bases occurs normally by surface tension.

\subsubsection{Example of support ball method}

Figure 18 presents an example of an application to a high-heat radiation package.[4]

An aluminum nitride (AlN) large heat spreader $45 \mathrm{~mm}$ square and $3 \mathrm{~mm}$ thick is set on a BGA with 359 pins. In this package, solder ball collapse can be avoided using support balls, even with heavy heat spreaders.

\subsection{Solder pouring method}

\subsubsection{Purpose}

In conventional soldering, the solder is set on the metal parts. Then the solder is heated and melted while using a supplemental material that removes the oxide films of solder such as those of flux.

However, in some cases, soldering with flux results in voids caused by flux remaining in the solder. Moreover, when using flux, the flux must be removed after soldering. 


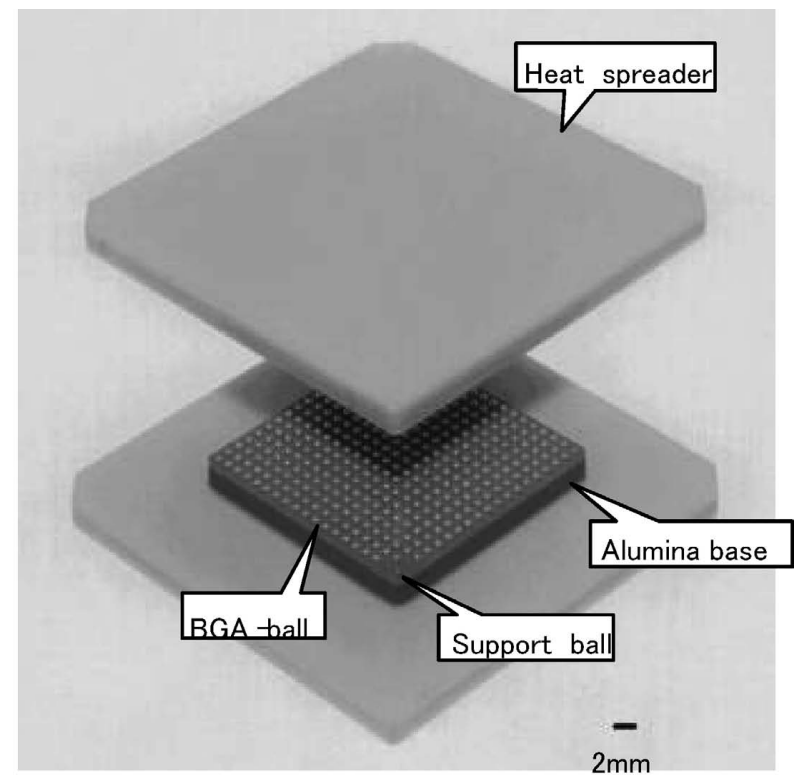

(a) Top and bottom views

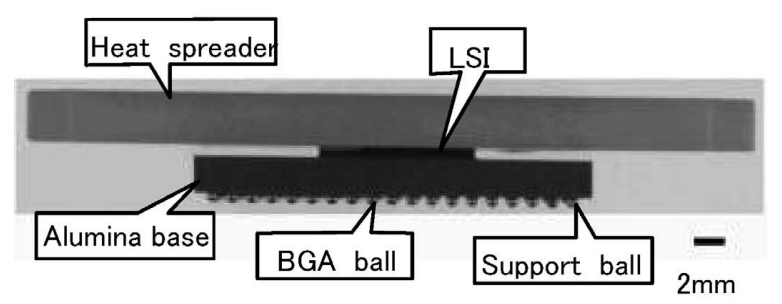

(b) Side view

Fig. 18 Top, bottom and side views of package.

When the soldering points are exposed, it is easy to remove flux after soldering. However, it is difficult to use flux for spots that are difficult to clean, such as internal parts of a package. Therefore, various fluxless methods are applied. However, it is difficult to maintain wettability for solder and metallizations compared with common, fluxbased, soldering methods. Therefore, management of processes tends to increase. In this study, the authors investigate methods that realize fluxless and voidless soldering by pouring solder to metal.

\subsubsection{Basic investigation of the solder pouring method}

As depicted in Figure 19, when plates with metallized areas for soldering are placed so that they face small gaps and pots with melted solder on the lower side, the solder gets wet and moves up. This is a capillary phenomenon by which solder wets with metallizations and the melted solder wets up to a narrow space.

Figure 20 presents illustrations of the experimental method. Metallized areas formed for soldering in ceramic plates $500 \mu \mathrm{m}$ wide and $15 \mathrm{~mm}$ long were then placed facing each other. These were stood on a small ceramic solder pot and observed.

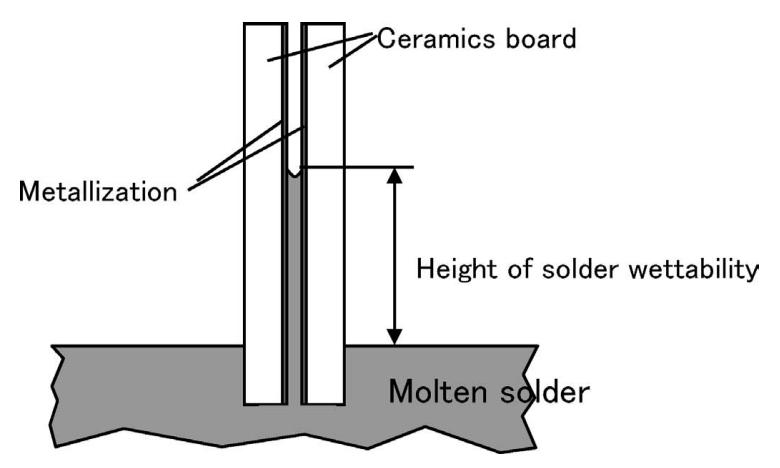

Fig. 19 Properties of molten solder.

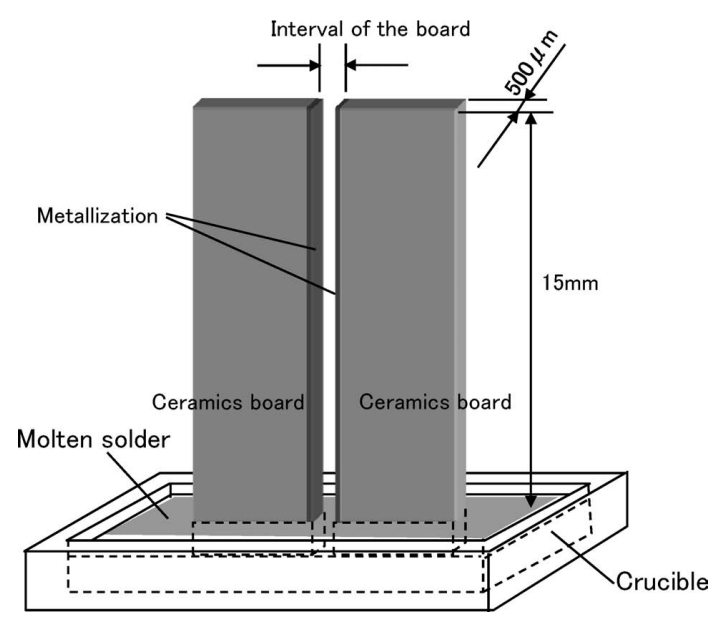

Fig. 20 Illustrations of the experimental method.

Table 2 Height of solder wettability.

\begin{tabular}{ccccc}
\hline & \multicolumn{4}{c}{ Interval of the board } \\
\cline { 2 - 5 } & $10 \mu \mathrm{m}$ & $60 \mu \mathrm{m}$ & $160 \mu \mathrm{m}$ & $260 \mu \mathrm{m}$ \\
\hline $\mathrm{Pb}-10 \mathrm{wt} \% \mathrm{Sn}$ & $100 \%$ & $100 \%$ & $100 \%$ & $100 \%$ \\
$\mathrm{Au}-20 \mathrm{wt} \% \mathrm{Sn}$ & $100 \%$ & $100 \%$ & $100 \%$ & $100 \%$ \\
\hline
\end{tabular}

Table 2 shows the observation results of the wet-up amount of the solder when the ceramic plate length was assumed as $100 \%$. The solder wetted up either solder material between the 15-mm-long plates for the metallizing interval from 10-260 $\mu \mathrm{m}$.

Figure 21 shows transmission X-ray observation images of the sample obtained using the solder pouring method invented in this study. The solder material used for the study was $\mathrm{Pb}-10 \mathrm{wt} \% \mathrm{Sn}$. Nitrogen was used for the reflow atmosphere. For comparison, the figure also shows transmission X-ray observation images of the sample obtained using a conventional method (a solder preform is set on metallizations of the parts; then reflow is performed).

The sample prototyped using the conventional method has numerous voids in an area of several hundred micrometers. In marked contrast, the sample obtained using the solder pouring method has almost no voids. The authors 


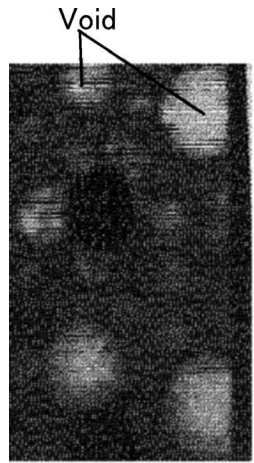

General method

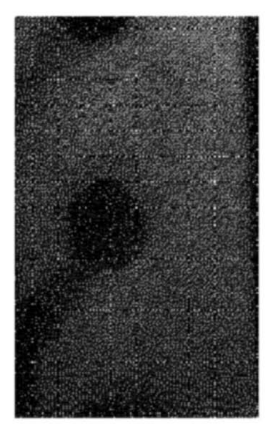

Pouring method
Void Fraction: 21\% Void Fraction: Less than 1\%

\section{$100 \mu \mathrm{m}$}

Fig. 21 Transmission X-ray image.

infer that this is because flux was not used for the process and little air mixed when the solder melted.

\section{Applied Technology}

This section presents descriptions of application examples of the technology introduced in Section 2 and 3.

\subsection{Application to a package for high-speed com- munications Part 1}

The support ball method is effective for heavy BGA structure packages. Figure 22 presents an application to a package for high-speed communications. [5, 6] An IC for high-speed transmissions has a BGA with 256 pins. Lowspeed signals are input and output from the BGA: highspeed signals of 43 Gbps are input and output from transmission line on the package surface through a large connector for maintaining quality. In this package, large connectors are set on two sides of the package. Therefore, the solder balls of the BGA collapse from those loads. This problem was resolved by using support balls, as shown in Section 3.1 .

\subsection{Application to a package for high-speed com- munication Part 2}

Another use of support balls is for positioning in a planar direction. Here, we introduce a case in which a thin lead is used as a high-speed transmission component. For example, as shown in Figure 23, when a transmission line formed for a lead is positioned on the transmission line on the package board for soldering, two component patterns are usually optically coupled for alignment. It is possible to perform positioning without special equipment using a support ball.

Figure 24 shows actual leads. Figure 25 shows the packages on which they are mounted.[7] Pads for support ball

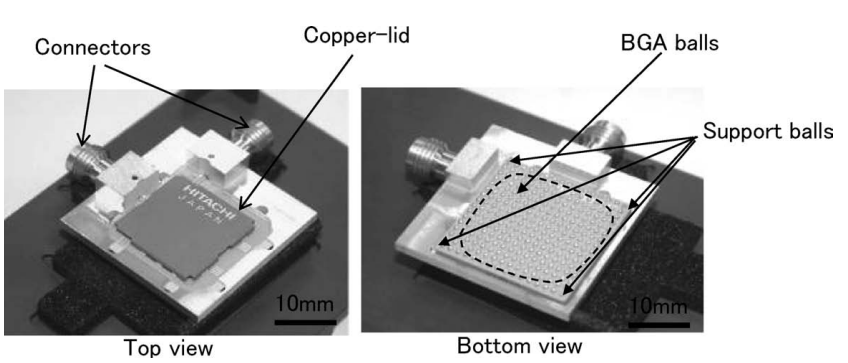

Fig. 22 Application of the semiconductor package for 43Gb/ s chipset.
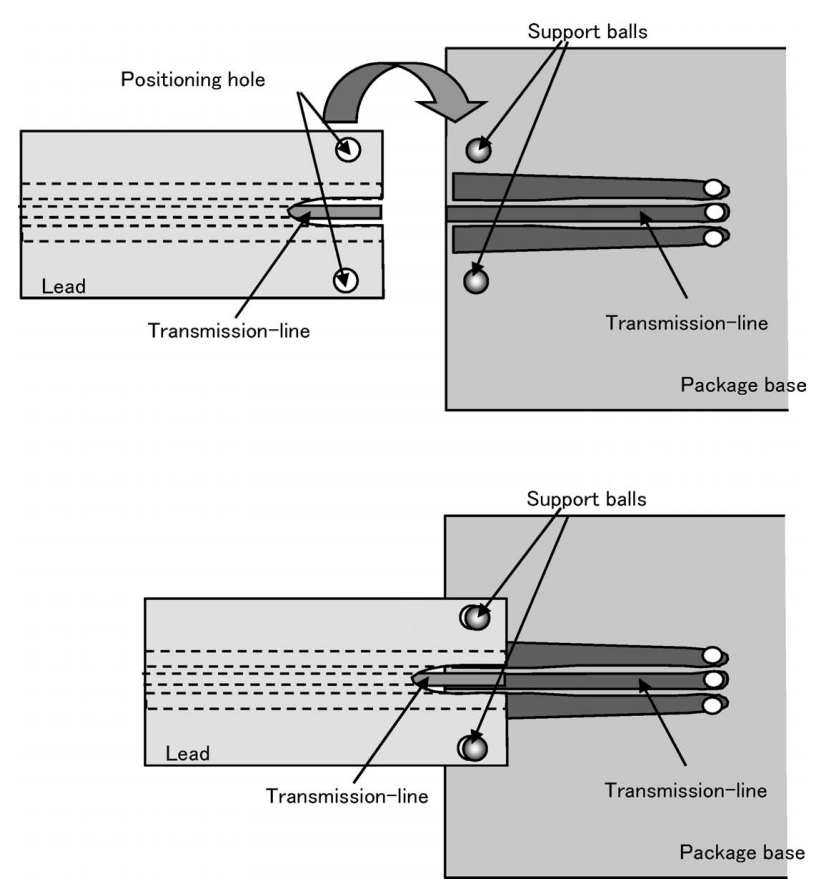

Fig. 23 Lead attachment method.

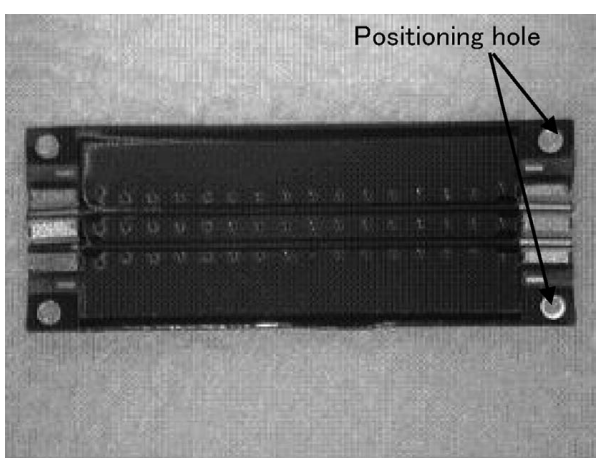

Fig. 24 (Reference) Lead part.

formation on the base side and transmission lines on the base side are formed in the same base manufacturing process. For that reason, the accuracy for each position is high.

Furthermore, the positioning hole of the leads and the transmission line of the leads are formed in the same process. Consequently, the accuracy for each position is high. By adding a positioning hole for the leads to the support balls on the base, high-accuracy mechanical positioning is 


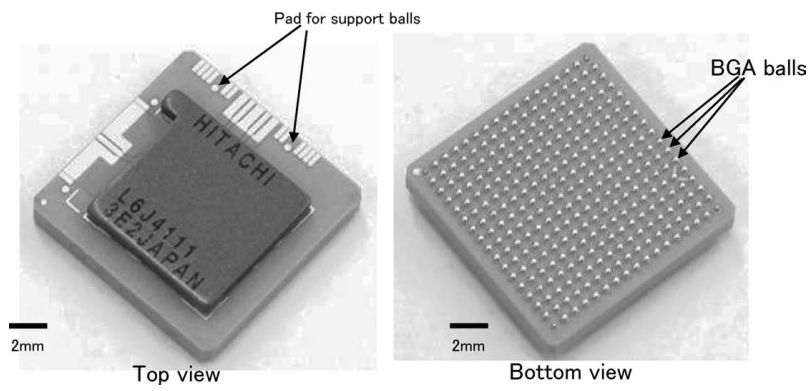

Fig. 25 Application of the semiconductor package using lead part.

realized for the transmission lines on the base and those of leads.

The authors confirmed that 43 Gbps signal transmission can be realized with this sample using a combination of a base and leads. Furthermore, the chip bump planarization shown in 2.1 was used for this package.

\subsection{Application to package sealing process}

High-quality hermetic sealing is realized by application of the solder pouring method described in 3.2 to a semiconductor package.

Figure 26 shows sealing process illustrations for the package sample used for the study. In a conventional sealing process, solder is set between a LSI chip and the cap and solder preforms are set on the sealing part between the cap and base for heating and melting.

For the solder pouring method, sufficient solder to fill the package sealing is poured between a cap and a LSI chip. Subsequently, the solder is poured into the package sealing part by heating and pressurizing the entire package. The poured solder is wetted and spread into the package sealing by wetting on metallizations and a capillary phenomenon. Then the gaps are filled with the solder. It is noteworthy that the solder flows only into the package sealing part and does not flow into the space between the LSI chip and base. When narrow gaps are filled up with solder, as described above, interactions of the spread of wetting onto a metallization and the capillary phenomenon are necessary. Because no metallization occurs for inducing solder on the outer circumference of an LSI chip, solder dose not flow into the space between the LSI chip and base.

Figure 27 portrays a transmission X-ray observation image of a sample for which package sealing was performed using the conventional process. The results show that several voids remain in the sealing part. These voids and fine shrinkage holes in the solder structures are connected; leakage occurs on the seal.

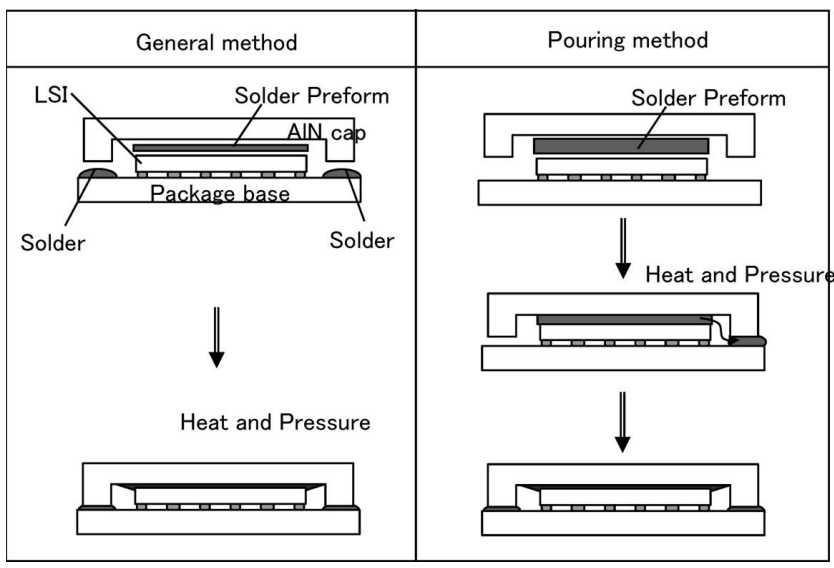

Fig. 26 Schematic of the seal process.

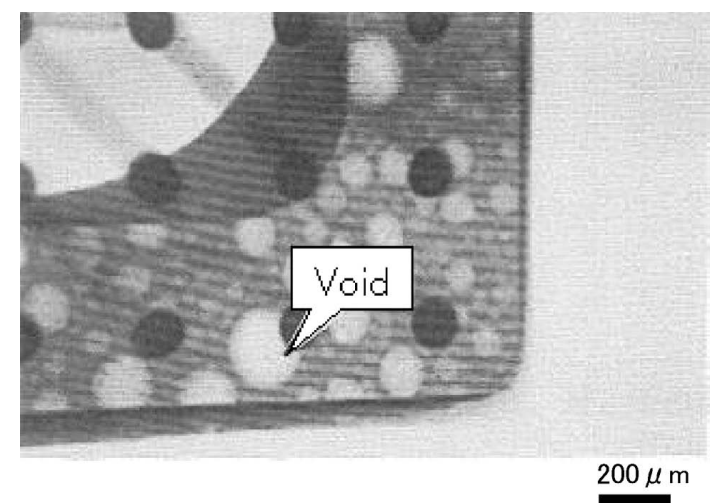

Fig. 27 Transmission X-ray image (General method).

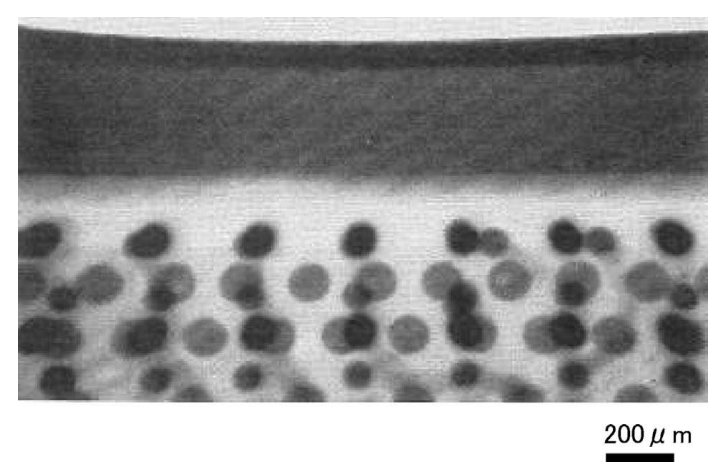

Fig. 28 Transmission X-ray image (Pouring method).

Figure 28 shows a transmission X-ray image of the sample obtained using the solder pouring method invented in this study. The results show that almost no voids are present in the sealing part. Furthermore, the solder bump planarization shown in 2.1 was used for this package.

\section{Conclusions}

Solder materials are used for electrodes or for sealing electronic parts. High-precision soldering and parts shape control can be realized using wetting on metallizations of these solder materials, de-wetting on parts other than metallizations, self-alignment and capillary phenomena in nar- 
row spaces, and so on.

As a result of plural process validations with actual samples, the authors have reached the following conclusions:

(1) This bump-transfer process produces solder bumps of uniform heights.

(2) This support-ball structure prevents BGA ball collapse. The authors demonstrated that the BGA structure is useful for heavy packages. The support ball method is effective for heavy BGA structure packages for high-speed communication.

(3) This solder-pouring method realizes fluxless and voidless sealing by pouring solder into a narrow space with metallization. Its effects were verified. The solder-pouring method is effective for highquality hermetic sealing packages.

\section{References}

[1] T. Sato, "Packaging Technology for SiP (System in Package) (<Special Articles $>$ Recent Jisso Technology for SiP and Its Future Trend)”, Journal of Japan Institute of Electronics Packaging Vol. 7, No. 2, pp. 111115, Mar. 2004.

[2] Y. Tsukada, K. Yamanaka and T. Nejime, "Current barriers and future direction of semiconductor pack- aging with flip chip bonding”, The IEICE transactions on electronics. C Vol. 91, No. 11, pp. 509-518, Nov. 2008.

[3] H. Kikuchi, T. Uda, T. Tanaka, T. Sato, T. Hayashida and M. Sakuma, "Solder Bump Transfer Material and Semiconductor Device Manufacturing Method (in Japanese)”, Japanese Patent 3267422 (2002).

[4] H. Ando, H. Kikuchi and T. Sato, "Flip-chip package with a heat spreader for high power dissipation LSI chips”, ECTC1999. 1999 Proceedings. 49 ${ }^{\text {th }}$, pp. 227231, 1999.

[5] T. Masuda, N. Shiramizu, K. Ohhata, K. Watanabe, T. Harada, H. Kikuchi and K. Washio, “A 43-Gb/s Low-jitter Output-circuit Design for 16:1 MUX IC Module Based on SiGe HBT Technology”, Vol. 103, No. 437, pp. 35-39, Nov. 2003.

[6] H. Ando, H. Kikuchi, S. Isomura and N. Nakazato, "Multi-chip BGA Package with the Connectors for 40Gb/s MUX/DMUX Chipset”, 2002 IEEE The $6^{\text {th }}$ VLSI Packaging Workshop of Japan. Technical Digest $5.2,2002$.

[7] H. Kikuchi, N. Nakazato, T. Suga, S. Isomura, T. Kubo and H. Sasaki, "Semiconductor Device and Electronic Device”, U.S.Patent 6,911,733. Jun. 28, 2005. 